Center for Quality and Productivity Improvement

UNIVERSITY OF WISCONSIN

610 Walnut Street

Madison, Wisconsin 53705

(608) 263-2520

(608) 263-1425 FAX

Report No. 124

\title{
The Use of Experimental Design and Multivariate Analysis in Product Improvement
}

\author{
M. R. Ellekjaer, M. A. Ilseng and T. Naes \\ January 1995
}

The Center for Quality and Productivity Improvement cares about your reactions to our reports. Please direct comments (general or specific) to: Report Editor, Center for Quality and Productivity Improvement, 610 Walnut Street, Madison, WI 53705; (608) 263-2520. All comments will be forwarded to the author(s). 


\title{
The Use of Experimental Design and Multivariate Analysis in Product Improvement
}

\author{
M. R. Ellekjaer \\ MATFORSK \\ Norwegian Food Research \\ Institute \\ Osloveien 1 \\ $N-1430$ ass \\ Norway
}

\author{
M. A. Ilseng \\ Norwegian Dairies \\ P. O. Box 153, \\ $N-2381$ \\ Brumunddal, \\ Norway
}

\author{
T. Naes \\ MATFORSK \\ Norwegian Food Research \\ Institute \\ Osloveien 1 \\ $N-1430$ ås \\ Norway
}

\begin{abstract}
The overall purpose of this study is to identify an effective strategy for improving the sensory quality of a product. A study on processed cheese was used to develop and illustrate our ideas. A screening experiment, with seven processing and ingredients variables, was performed in order to identify the processing variables with the greatest effect on sensory quality. A fractional factorial design with resolution IV was used to keep the number of experimental runs to a minimum. ANOVA and normal plots were used to evaluate the effects of the different factors on the sensory variables one by one. The same factors were identified as being important when the scores from a principal component analysis (PCA) of the sensory variables were analyzed. PCA was found to be of value in identifying samples that had improved properties compared to today's product in addition to having a low intensity of undesirable properties.
\end{abstract}

KEYWORDS: Fractional factorial design; Multi-response data; Principal component analysis (PCA). 


\title{
The Use of Experimental Design and Multivariate Analysis in Product Improvement
}

\author{
M. R. Ellekjaer, M. A. Ilseng and T. Naes
}

\begin{abstract}
The overall purpose of this study is to identify an effective strategy for improving the sensory quality of a product. A study on processed cheese was used to develop and illustrate our ideas. A screening experiment, with seven processing and ingredients variables, was performed in order to identify the processing variables with greatest effect on the sensory quality. A fractional factorial design with resolution IV was used to keep the number of experimental runs to a minimum. ANOVA and normal plots were used to evaluate the effects of the different factors on the sensory variables one by one. The same factors were identified as being important when the scores from a principal component analysis (PCA) of the sensory variables were analyzed. PCA was found to be of value in identifying samples that had improved properties compared to today's product in addition to having a low intensity of undesirable properties.
\end{abstract}

\section{INTRODUCTION}

In order to improve the quality of an existing food product it is of importance to identify those ingredients and processing variables (here called factors) which have the greatest effect on the quality. The quality of the product should then be optimized on the basis of these factors.

How could these ingredients and processing conditions be identified? An unsophisticated approach is to 'hold everything constant and only vary one factor at the time'. This approach provides an estimate of the effect of a single factor at selected fixed conditions of the other factors. However, for such an estimate to have general relevance it is necessary to assume no interaction effects.

The use of statistical methods for experimental design make it possible to vary several factors at the same time with the use of a minimum of experiments (Box et al., 1978). This approach has several advantages over the one factor at the time approach. The key advantages are fewer experimental runs, increased precision, possibility of detecting interaction effects and a wider inductive basis (Box et al., 1978; Box et al., 1990). The use of experimental design is thus an effective approach to identify the most significant factors regarding the quality of a product. It can contribute to optimize the quality of the product to fulfill the customers needs in a costefficient way. Such techniques have primarily been used when optimizing products for the different response variables separately. Food however, is multivariate in nature and the sensory quality of the product is a combination of taste, smell, texture and appearance attributes. The optimal quality of the sensory attributes which describe the most relevant sensory variation have to be defined in order to develop a product with the best possible sensory quality. Multivariate methods, in particular principal component analysis (PCA), is an important tool for identifying these sensory attributes. The combination of experimental design and multivariate methods should therefore be a powerful tool for improving the process of product development. Carlson et al. (1992) discussed strategies for analyzing screening experiments when many response variables had been measured. They demonstrated that principal component (PC) decomposition of the responses could be applied to evaluate screening experiments with multiple responses. The score vectors which relate the individual experiments (samples) to each other, could be used as response variables to identify significant factors. The loading vectors could be used to identify both the response variables describing the main variation and response variables describing the same phenomena among the samples.

The overall purpose of the study was to identify an effective and goal oriented strategy for improving the sensory quality of a product. Improvement of the texture properties of a commercially processed cheese was used as an example. This cheese got low scores in a consumer test of processed cheeses from different companies (unpublished results). Sensory analysis of the same cheeses revealed that this cheese 
had lower intensity for meltability and was more sticky, grainy and adhesive than the preferred cheese.

In order to improve the texture properties we wanted to identify both the ingredients and processing variables (factors) with the largest effect on the sensory quality of processed cheese, and the processing conditions which resulted in improved sensory quality compared to the current product. The results obtained when using the traditional approach of evaluating the effect of the different factors on each sensory attribute separately will be compared with the results obtained by using the multivariate approach, which uses the scores of the main principal components (after PCA of sensory data) as response variables.

\section{MATERIAL AND METHODS}

\section{THE EXPERIMENT}

The effect of seven different ingredients or processing factors on the sensory quality of processed cheese was studied. These were type of melting salt (A), maturity of cheese (B), dry matter of processed cheese (C), pH (D), addition of dry matter (E), after cooling (F) and cooling of processed cheese (G). Two different levels were selected for each factor. These are listed in Table 1 . A $2^{6-2}$ fractional factorial design was set up for each melting salt, resulting in 32 different experimental runs. The design has resolution IV, which means that the main effects and the twofactor interactions were not confounded with one another whereas the two-factor interactions were confounded (Box et al., 1978). The confounding pattern of the two-factor interactions was as follows: $\mathrm{BC}=\mathrm{FG}, \mathrm{BD}=\mathrm{EG}, \mathrm{CD}=\mathrm{EF}, \mathrm{BE}=\mathrm{DG}, \mathrm{CE}=\mathrm{DF}$, $\mathrm{DE}=\mathrm{CF}=\mathrm{BG}$ and $\mathrm{BF}=\mathrm{CG}$. The three- and four-factor interactions were confounded with the main effects and two-factor interactions.

Three replicates of the center point for each melting salt were made in order to estimate the experimental error. These replicates were used in combination with three productions of a reference sample made from the original recipe. The sample identifications and the processing conditions used are presented in Table 1. All the experimental runs were produced in randomized order.

\section{SENSORY EVALUATION}

The sensory attributes of the processed cheese were evaluated by a sensory panel. The sensory attributes evaluated were glossiness, ability to retain shape, adhesiveness, firmness, graininess, stickiness, meltability, taste of cheese, saltiness, bitterness, condensation and off-flavor. The processed cheeses were analyzed in two sensory replicates and six to eight assessors took part at each evaluation.

Table 1. Identification of samples and processing conditions used. Processed cheese produced using original recipe is marked with *

\begin{tabular}{|c|c|c|c|c|c|c|c|}
\hline ID & $\begin{array}{c}\text { Melting } \\
\text { salt }\end{array}$ & $\begin{array}{l}\text { Maturity } \\
\text { of cheese } \\
\text { (months) }\end{array}$ & $\begin{array}{c}\text { Dry } \\
\text { matter of } \\
\text { processed } \\
\text { cheese } \\
(\%)\end{array}$ & $\mathrm{pH}$ & $\begin{array}{l}\text { Addition } \\
\text { of dry } \\
\text { matter } \\
\text { (क) }\end{array}$ & $\begin{array}{c}\text { After } \\
\text { creaming }\end{array}$ & $\begin{array}{c}\text { Cooling } \\
\text { of } \\
\text { processed } \\
\text { cheese }\end{array}$ \\
\hline $\begin{array}{l}\text { A1 } \\
\text { A2 } \\
\text { A3 } \\
\text { A4 } \\
\text { A5 } \\
\text { A6 } \\
\text { A7 } \\
\text { A8 } \\
\text { A9 } \\
\text { A10 } \\
\text { A11 } \\
\text { A12 } \\
\text { A13 } \\
\text { A14 } \\
\text { A15 } \\
\text { A16 } \\
\text { B1 } \\
\text { B2 } \\
\text { B3 } \\
\text { B4 } \\
\text { B5 } \\
\text { B6 } \\
\text { B7 } \\
\text { B8 } \\
\text { B9 } \\
\text { B10 } \\
\text { B11 } \\
\text { B12 } \\
\text { B13 } \\
\text { B14 } \\
\text { B15 } \\
\text { B16 } \\
\text { B0 } \\
\text { B0 } \\
\text { B0 } \\
\text { A0 } \\
\text { A0 } \\
\text { A0 } \\
* \\
* \\
* \\
\text { * }\end{array}$ & $\begin{array}{l}\text { A } \\
\text { A } \\
\text { A } \\
\text { A } \\
\text { A } \\
\text { A } \\
\text { A } \\
\text { A } \\
\text { A } \\
\text { A } \\
\text { A } \\
\text { A } \\
\text { A } \\
\text { A } \\
\text { A } \\
\text { A } \\
\text { B } \\
\text { B } \\
\text { B } \\
\text { B } \\
\text { B } \\
\text { B } \\
\text { B } \\
\text { B } \\
\text { B } \\
\text { B } \\
\text { B } \\
\text { B } \\
\text { B } \\
\text { B } \\
\text { B } \\
\text { B } \\
\text { B } \\
\text { B } \\
\text { B } \\
\text { A } \\
\text { A } \\
\text { A } \\
\text { A } \\
\text { A } \\
\text { A }\end{array}$ & 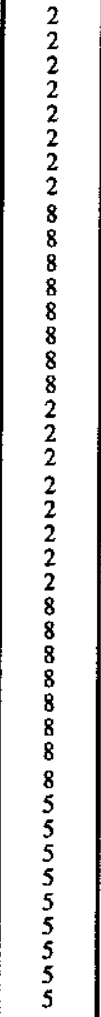 & $\begin{array}{l}36 \\
36 \\
36 \\
36 \\
40 \\
40 \\
40 \\
40 \\
36 \\
36 \\
36 \\
36 \\
40 \\
40 \\
40 \\
40 \\
36 \\
36 \\
36 \\
36 \\
40 \\
40 \\
40 \\
40 \\
36 \\
36 \\
36 \\
36 \\
40 \\
40\end{array}$ & $\begin{array}{l}5.7 \\
5.7 \\
6.1 \\
6.1 \\
5.7 \\
5.7 \\
6.1 \\
6.1 \\
5.7 \\
5.7 \\
6.1 \\
6.1 \\
5.7 \\
5.7 \\
6.1 \\
6.1 \\
5.7 \\
5.7 \\
6.1 \\
6.1 \\
5.7 \\
5.7 \\
6.1 \\
6.1 \\
5.7 \\
5.7 \\
6.1 \\
6.1 \\
5.7 \\
5.7 \\
6.1 \\
6.1 \\
5.9 \\
5.9 \\
5.9 \\
5.9 \\
5.9 \\
5.9 \\
5.9 \\
5.9 \\
5.9\end{array}$ & $\begin{array}{c}0 \\
3 \\
0 \\
3 \\
0 \\
3 \\
0 \\
3 \\
0 \\
3 \\
0 \\
3 \\
0 \\
3 \\
0 \\
3 \\
0 \\
3 \\
0 \\
3 \\
0 \\
3 \\
0 \\
3 \\
0 \\
3 \\
0 \\
3 \\
0 \\
3 \\
0 \\
3 \\
1.5 \\
1.5 \\
1.5 \\
1.5 \\
1.5 \\
1.5 \\
0 \\
0 \\
0\end{array}$ & $\begin{array}{l}15 \\
45 \\
45 \\
15 \\
45 \\
15 \\
15 \\
45 \\
15 \\
45 \\
45 \\
15 \\
45 \\
15 \\
15 \\
45 \\
15 \\
45 \\
45 \\
15 \\
45 \\
15 \\
15 \\
45 \\
15 \\
45 \\
45 \\
15\end{array}$ & $\begin{array}{l}\text { slow } \\
\text { rapid } \\
\text { rapid } \\
\text { slow } \\
\text { slow } \\
\text { rapid } \\
\text { rapid } \\
\text { slow } \\
\text { rapid } \\
\text { slow } \\
\text { slow } \\
\text { rapid } \\
\text { rapid } \\
\text { slow } \\
\text { slow } \\
\text { rapid } \\
\text { slow } \\
\text { rapid } \\
\text { rapid } \\
\text { slow } \\
\text { slow } \\
\text { rapid } \\
\text { rapid } \\
\text { slow } \\
\text { rapid } \\
\text { slow } \\
\text { slow } \\
\text { rapid } \\
\text { rapid } \\
\text { slow } \\
\text { slow } \\
\text { rapid } \\
\text { medium } \\
\text { medium } \\
\text { medium } \\
\text { medium } \\
\text { medium } \\
\text { medium } \\
\text { medium } \\
\text { medium } \\
\text { medium }\end{array}$ \\
\hline
\end{tabular}

\section{DATA ANALYSIS}

Analysis of variance (ANOVA) was performed in order to identify the sensory attributes which had more variation between samples than could be attributed to measurement variation. A crossclassification model with the effects of samples, assessors and the two-factor interaction between samples and assessors was applied, where assessors and two-factor interactions were evaluated as random effects. ANOVA was performed by the GLM procedure in the SAS system (SAS Institute, Cary, NC). The GLM module computes expected mean 
squares and use these expectations to find combinations of mean squares that give approximate F-tests even for unbalanced designs.

The average response over assessors and sensory replicates for each sample of the significant sensory attributes was used in the further analysis (Table 2).

Principal component analysis (PCA) (Mardia et al., 1979) was performed on the significant sensory attributes identified by ANOVA. PCA treats all response variables (sensory attributes) simultaneously. The data are modeled in terms of a few significant principal components (PC). The principal components contain the main phenomena or systematic variability present in the data.

The use of scale vary for the different sensory attributes . In order to equalize the chances for the different attributes to contribute to the model the sensory attributes were standardized to equal variance and mean centered prior to PCA (Baardseth et al., 1992). Non-significant attributes (identified by

Table 2. The average response of the samples for the different sensory attributes.

\begin{tabular}{|c|c|c|c|c|c|c|c|c|}
\hline $\begin{array}{l}\text { sample } \\
\text { number }\end{array}$ & Glossiness & $\begin{array}{l}\text { Ability to } \\
\text { retain } \\
\text { shape }\end{array}$ & Adhesiveness & Firmness & Graininess & Stickiness & Meltability & Condensation \\
\hline A1 & 7.08 & 7.08 & 4.15 & 4.46 & 3.77 & 3.15 & 7.23 & 1.85 \\
\hline $\mathrm{A} 2$ & 7.07 & 5.21 & 4.43 & 3.43 & 4.36 & 2.86 & 7.36 & 1.79 \\
\hline $\mathrm{A} 3$ & 7.69 & 1.38 & 5.00 & 1.54 & 2.15 & 3.00 & 7.92 & 2.62 \\
\hline A4 & 7.69 & 1.85 & 6.08 & 1.92 & 2.77 & 3.92 & 6.92 & 2.69 \\
\hline A5 & 6.47 & 6.87 & 5.27 & 5.40 & 5.53 & 5.13 & 5.20 & 2.53 \\
\hline A6 & 6.07 & 7.79 & 4.79 & 6.21 & 5.07 & 5.00 & 5.14 & 2.00 \\
\hline A7 & 6.08 & 8.08 & 5.15 & 8.00 & 3.62 & 6.23 & 3.54 & 1.46 \\
\hline A8 & 6.14 & 6.21 & 6.29 & 5.36 & 6.07 & 5.43 & 4.50 & 1.86 \\
\hline A9 & 7.29 & 7.50 & 3.71 & 4.71 & 2.21 & 2.93 & 7.64 & 2.50 \\
\hline A10 & 7.00 & 7.50 & 4.07 & 5.43 & 4.00 & 3.71 & 6.36 & 1.43 \\
\hline A11 & 7.20 & 6.67 & 4.20 & 4.47 & 4.47 & 3.73 & 6.47 & 1.67 \\
\hline A12 & 7.29 & 4.64 & 4.79 & 3.14 & 3.79 & 3.00 & 7.36 & 1.57 \\
\hline A13 & 6.47 & 8.40 & 4.60 & 6.87 & 4.80 & 4.93 & 5.33 & 1.73 \\
\hline A14 & 6.64 & 8.64 & 4.79 & 7.93 & 3.07 & 5.71 & 4.43 & 2.00 \\
\hline A15 & 5.93 & 8.29 & 3.93 & 7.86 & 6.29 & 5.50 & 4.43 & 1.43 \\
\hline A16 & 6.08 & 8.08 & 3.77 & 7.38 & 3.77 & 5.69 & 4.62 & 1.92 \\
\hline B1 & 6.62 & 7.00 & 4.08 & 4.62 & 4.00 & 2.77 & 7.46 & 2.00 \\
\hline B2 & 6.80 & 4.87 & 4.93 & 3.53 & 5.40 & 3.13 & 6.67 & 2.20 \\
\hline B3 & 7.57 & 1.36 & 4.86 & 1.07 & 3.64 & 2.86 & 7.86 & 2.93 \\
\hline B4 & 7.29 & 3.00 & 4.71 & 2.57 & 2.93 & 3.43 & 7.07 & 2.50 \\
\hline B5 & 6.47 & 7.60 & 4.53 & 5.40 & 5.87 & 4.80 & 5.73 & 2.13 \\
\hline B6 & 7.08 & 6.62 & 4.46 & 5.15 & 4.23 & 4.31 & 6.23 & 2.62 \\
\hline B7 & 6.14 & 8.00 & 6.00 & 6.79 & 6.00 & 5.79 & 4.57 & 1.86 \\
\hline B8 & 6.54 & 6.15 & 5.69 & 4.23 & 2.62 & 5.00 & 5.23 & 2.46 \\
\hline B9 & 7.38 & 8.31 & 3.38 & 5.15 & 3.08 & 3.62 & 6.31 & 1.69 \\
\hline B10 & 6.67 & 8.20 & 4.07 & 5.67 & 3.87 & 3.47 & 6.60 & 1.73 \\
\hline B 11 & 7.50 & 2.71 & 4.36 & 2.29 & 4.21 & 2.93 & 7.64 & 3.07 \\
\hline B 12 & 7.36 & 4.57 & 4.57 & 3.29 & 4.50 & 2.93 & 7.29 & 1.71 \\
\hline B 13 & 6.29 & 8.36 & 3.79 & 5.93 & 6.14 & 4.14 & 5.71 & 1.93 \\
\hline B14 & 7.54 & 8.31 & 4.08 & 6.46 & 3.46 & 4.54 & 4.85 & 2.00 \\
\hline B15 & 5.47 & 8.33 & 3.80 & 8.00 & 6.13 & 5.87 & 3.73 & 1.40 \\
\hline B16 & 6.69 & 7.62 & 5.00 & 5.23 & 3.38 & 4.46 & 5.54 & 1.62 \\
\hline B0 & 6.08 & 7.46 & 4.85 & 6.38 & 4.00 & 5.00 & 5.77 & 1.77 \\
\hline B0 & 5.79 & 6.43 & 4.64 & 4.57 & 5.29 & 4.21 & 6.21 & 1.64 \\
\hline Bo & 6.92 & 6.54 & 6.46 & 5.38 & 2.38 & 5.77 & 5.00 & 1.85 \\
\hline $\mathrm{AO}$ & 5.46 & 8.15 & 3.69 & 7.00 & 4.31 & 5.38 & 4.77 & 1.85 \\
\hline AO & 6.40 & 6.67 & 5.47 & 5.00 & 5.73 & 4.60 & 5.93 & 1.80 \\
\hline $\mathrm{AO}$ & 6.14 & 7.21 & 5.29 & 5.36 & 5.50 & 3.64 & 5.36 & 1.43 \\
\hline$*$ & 6.93 & 6.93 & 4.71 & 4.71 & 2.86 & 4.21 & 6.64 & 2.64 \\
\hline * & 6.64 & 7.71 & 4.21 & 5.79 & 3.50 & 4.43 & 5.93 & 1.29 \\
\hline$*$ & 6.62 & 7.85 & 4.85 & 5.38 & 2.92 & 4.85 & 5.23 & 1.54 \\
\hline
\end{tabular}


ANOVA) were removed prior to standardization to avoid standardization of meaningless variables.

Full cross-validation (Martens and Næs, 1989) was used to validate the PCA model. Unscrambler Version 5.0 software package (Camo a/S, Trondheim, Norway) was used for PCA.

The effect of the different factors was estimated using each single attribute as a response and using scores from the first three principal components (PC) as responses. The significance of the main and twofactor interaction effects was determined by including both the three samples produced in triplicates (reference + center samples) and three and higher order interactions for estimating error terms (Table 3 and 4).

Normal probability plotting (Box et al., 1978) was also used to identify the significant main and two-factor interaction effects. This method is used for Figure 1, 2a, 2b, 2c. The normal plots contain similar information as the significance tables described above, but were incorporated because they represent an easier way of visualizing the information.

Table 3. Overview of significant main effects and two-factor interactions when evaluating each sensory attribute separately. ${ }^{* * *}=p<0.001, * *=p<0.01, *=p<0.05$

\begin{tabular}{|c|c|c|c|c|c|c|c|c|}
\hline & Glossiness & $\begin{array}{l}\text { Ability to } \\
\text { retain shape }\end{array}$ & Adhesivness & Firmness & Graininess & Stickiness & Meltiness & Condensation \\
\hline $\begin{array}{l}\text { A -Melting salt } \\
\text { B - Maturity of cheese } \\
\text { C - Dry matter of processed cheese } \\
\text { D - pH } \\
\text { E - Addition of dry matter } \\
\text { F - After creaming } \\
\text { G - Cooling of processed cheese } \\
\text { A*B } \\
A^{*} C \\
B^{*} C=F^{*} G \\
A^{*} D \\
B^{*} D=E * G \\
C * D=E^{*} F \\
A^{*} E \\
B^{*} E=D * G \\
C^{*} E=D^{*} F \\
D^{*} E=C^{*} F=B^{*} G \\
A^{*} F \\
B^{*} F=C^{*} G \\
A^{*} G\end{array}$ & $-0.84^{* * *}$ & $\begin{array}{c}1.69^{* * *} \\
2.59^{* * *} \\
-1.96^{* * *} \\
-0.68 *\end{array}$ & $\begin{array}{c}-0.85^{* *} \\
0.57^{*}\end{array}$ & $\begin{array}{c}-0.55 * \\
1.26^{* * *} \\
2.81^{* * *} \\
-0.83^{* *} \\
-0.82^{* *} \\
1.26^{* * *}\end{array}$ & $1.06 * *$ & $1.94^{* * *}$ & $-2.21 * * *$ & $-0.38 *$ \\
\hline
\end{tabular}

Table 4. Overview of significant main effects and two-factor interactions when using the score from the three first principal components $(P C)$

\begin{tabular}{|c|c|c|c|}
\hline & PCI & $\mathrm{PC} 2$ & $P C 3$ \\
\hline $\begin{array}{l}\text { A - Melting salt } \\
\text { B - Marvity of cheese } \\
\text { C- Dry matter of processed cheese } \\
\text { D - pH } \\
\text { E - Addition of dry matter } \\
\text { F- After creaning } \\
\text { G - C Cooling of processed cheese }\end{array}$ & $\begin{array}{l}-1.05 * * \\
-3.64 * * *\end{array}$ & $\begin{array}{c}-1.40 * * * \\
0.90 * * \\
1.14 * *\end{array}$ & \\
\hline $\begin{array}{l}\mathrm{A}^{*} \mathrm{~B} \\
\mathrm{~A}^{*} \mathrm{C} \\
\mathrm{A}^{*} \mathrm{C}=\mathrm{F}^{*} \mathrm{G} \\
\mathrm{A}^{*} \mathrm{D} \\
\mathrm{A}^{*} \mathrm{D}=\mathrm{E}^{*} \mathrm{G} \\
\mathrm{C}^{*} \mathrm{D}=\mathrm{E}^{*} \mathrm{~F}\end{array}$ & $-1.63 * * *$ & & $0.79 * *$ \\
\hline $\begin{array}{l}A^{*} E \\
B^{*} E=D^{*} G \\
C^{*}=D^{*} F \\
D^{*}=-C^{*} F=B^{*} G \\
A^{*} F \\
B^{*} F=C^{*} G \\
A^{*} G=\end{array}$ & $0.94 *$ & & $0.78 * *$ \\
\hline
\end{tabular}

\section{RESULTS}

\section{SIGNIFICANT SENSORY ATTRIBUTES}

With the use of ANOVA it was determined that the sensory attributes glossiness, ability to retain shape, adhesiveness, firmness, graininess, stickiness, meltability and condensation described significant differences $(p<0.05)$ between the samples. Taste of cheese, saltiness, bitterness and off-flavor did not describe significant differences between samples and were therefore removed before further analysis. In Table 2 the average response for each sample are presented for the significant sensory attributes. 


\section{EVALUATION OF EFFECTS FOR ONE RESPONSE VARIABLE AT A TIME}

Analyzing the effects of the different factors for each sensory attribute separately revealed that the dry matter content of processed cheese had a significant effect on the majority of the sensory attributes (Table 3 ). $\mathrm{pH}$ and grade of maturity also had substantial effect on a number of the sensory attributes. All three factors affected in particular the firmness and ability to retain shape. The dry matter content had in addition effect on the glossiness, stickiness, meltability and graininess of the processed cheeses.

A visual validation, by using normal probability plot, of factors with significant effects on each sensory attribute results in 8 separate plots as the number of plots equals the number of response variables. In Figure 1, only the normal probability plot for the attribute ability to retain shape which shows four significant effects, is presented. The ability to retain shape increased both with increasing amount of dry matter content $(C)$ in processed cheese and increasing grade of maturity of the cheese (B) and decreased with increasing $\mathrm{pH}$ (D). In addition there was a significant interaction effect between $C^{*} \mathrm{D}+\mathrm{E}^{*} \mathrm{~F}$. Since both $\mathrm{C}$ (dry matter content) and $\mathrm{D}$ $(\mathrm{pH})$ have significant main effects for most of the same responses, the significant 2-factor interaction $C * D+E^{*} F$ is probably mainly an interaction between $\mathrm{C}$ and D. From Table 3 we can see that there was a significant 2-factor interaction $C * D+E^{*} F$ for a number of attributes; ability to retain shape, firmness, glossiness, meltability and condensation.

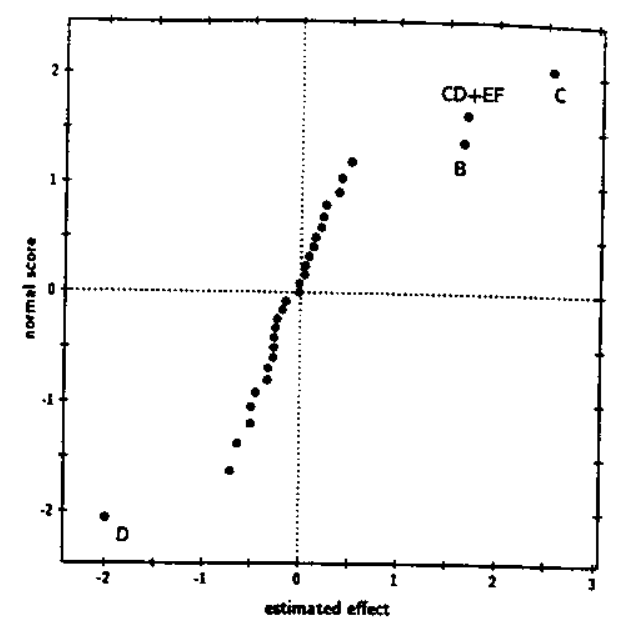

Figure 1. Normal probability plot for ability to retain shape.

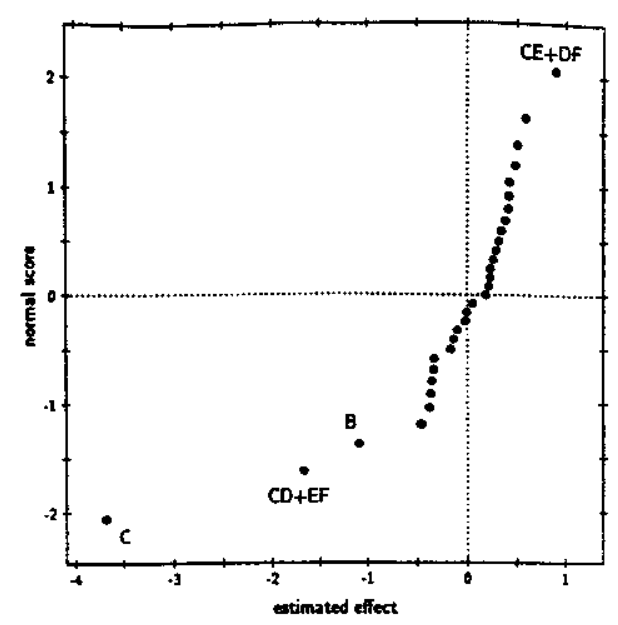

Figure 2a. Normal probability plot for Princinal Component $(P C) 1$

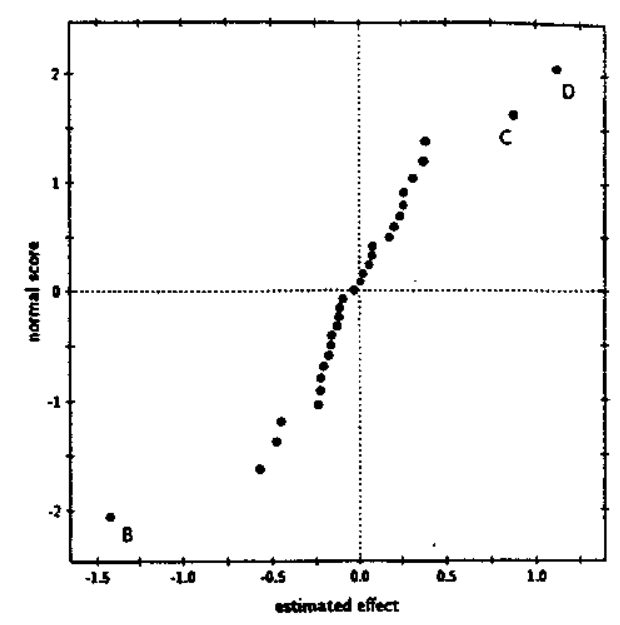

Figure 2b. Normal probability plot for $P C ?$

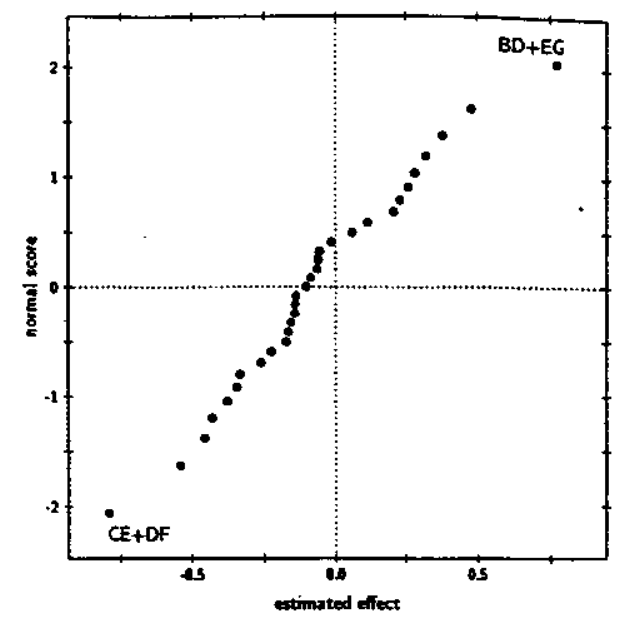

Figure 2c. Normal probability plot for $\mathrm{PC} 3$ 


\section{EVALUATION OF EFFECTS USING PC-SCORES AS RESPONSE VARIABLES}

The three first PC's obtained by PCA, described $74.8 \%$ of the sensory variation among the samples for processed cheese. The scores and loadings for the first 2 PC's are presented in Figure 3.

The first two PC's in Figure 2 visualize the main variation (64.9\% of the variation) among the samples (score plot) and the sensory attributes that contribute to describe this variation (loading plot). The attributes with high positive or high negative loadings describe the main variation among the samples. These were firmness, ability to retain shape, stickiness, meltiness and glossiness. Samples with high positive scores at the first PC had high intensity of meltiness and glossiness and low intensity of firmness, ability to retain shape and stickiness. The second PC described mainly variation among the samples related to adhesiveness (76\% of this variation was described by PC2). Samples with high positive scores had high intensity of adhesiveness, whereas samples with high negative scores had low intensity of this property.

If one has a reference sample as we had in this case, this plot can be used for identifying samples with improved sensory quality compared to the reference sample. The ultimate goal of the present experiment was to produce a cheese which was less

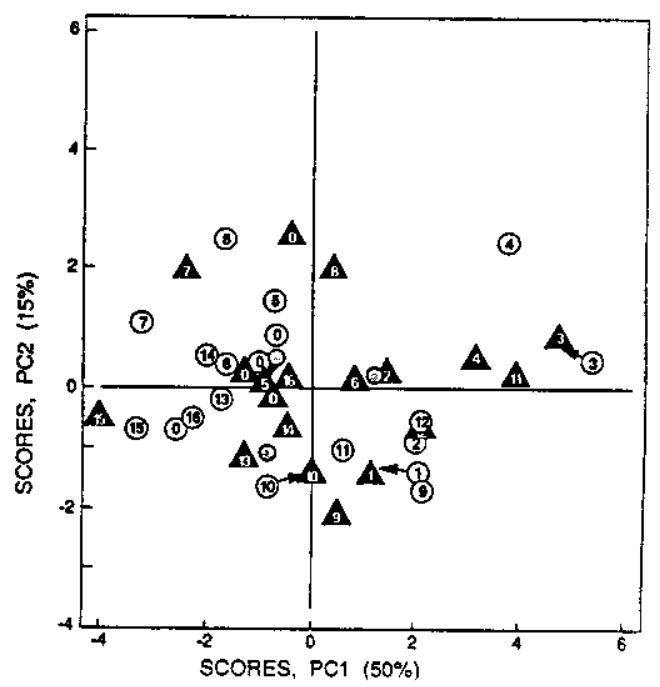

Figure 3a. sticky, grainy and adhesive, with higher intensity for meltability and still was able to keep the shape. By looking at the plots in Figure 3 we can see that samples in the lower right corner of the score plot, with both positive scores at the first PC and high negative scores at the second $\mathrm{PC}$, had these properties. These were samples B1, B9, B10, A1, A9 and $\mathrm{A} 10$ which were produced with low content of dry matter and with lowest $\mathrm{pH}$. These samples were looser and more glossy than the cheese produced according to today's specifications. In addition, they were less sticky, grainy and adhesive, and had the ability to retain the shape.

In Figure 4 the scores and loadings for PC2 versus PC3 are plotted. These two PC described respectively, $14.9 \%$ and $9.9 \%$ of the variation among the samples. This variation was related to the unwanted properties adhesiveness and graininess, of processed cheese. Samples with high intensity of adhesiveness had high positive scores at $\mathrm{PC} 2$, whereas samples with high intensity of graininess had high positive scores at PC3. This plot could therefore be used to identify the preferred samples (identified in Figure 3 by plotting PC1 against PC2) with low intensity of both adhesiveness and graininess. Such samples should have high negative scores at both PC2 and PC3. One can see that the two "9 samples" which were among the preferred samples in Figure 3,

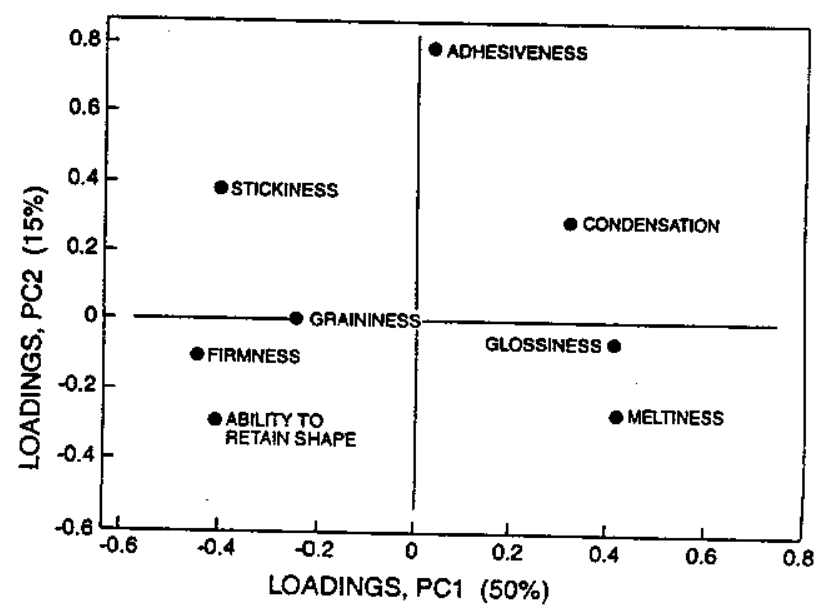

Figure 3b.

Figure 3a and 3b. Principal component analysis (PCA) of the sensory data of processed cheese. PCA scores and loadings for principal component $I$ and 2 (PC 1 and PC 2). The open circles represent processed cheese produced with melting salt $A$; The triangles represent processed cheese produced with melting salt $B$. Processed cheese produced after today's specifications are indicated by filled circles. 
had these properties. These samples were made with different melting salt but were produced at similar conditions. These were: Grade of maturity (B): 8 months, dry matter content (C): $36 \%$ pH (D):5.7, addition of dry matter $(\mathrm{E}): 0 \%$, after creaming $(\mathrm{F}): 15$ minutes and cooling of processed cheese (G): rapid.

The effects of the different factors on the three first PC were also evaluated. The first principal component which described $50.0 \%$ of the variation among the samples, described mainly variation among the samples related to firmness and meltability, but also glossiness, ability to retain shape and stickiness were related to this PC direction. It appeared that dry matter content came out with the greatest effect, but also grade of maturity and twofactor interaction between $\mathrm{pH}$ and dry matter content had significant effect on this variation (Table 4). This is visualized in a normal probability plot in Figure $2 \mathrm{a}$. These results are in accordance with the main results when evaluating the significant effects for one sensory attribute separately (see Table 3 ).

Analyzing the effects of the different factors on the variation among the samples for the second PC which described $75.6 \%$ of the variation in adhesiveness, revealed that both the maturity and dry matter content of the processed cheese in addition to $\mathrm{pH}$ had significant effects (Table 4 and Figure $2 \mathrm{~b}$ ). This is in accordance with the significant main effects identified when evaluating adhesiveness separately (see Table 3), except that dry matter of the processed cheese was not identified as significant in that case. There were no significant main effects on the variation among the samples at the third PC which was mainly related to the graininess of these samples (Table 4 and Figure 2c). Only two-factor interaction effects were identified having significant effect on the variation among the samples at the third $\mathrm{PC}$, and these were the same interaction effects as when evaluating the effects on graininess separately (see Table 3).

\section{DISCUSSION}

Evaluation of the effects of the different factors was done by both using the scores from the 3-first PC and the 8 significant sensory attributes, as response variables. ANOVA on the scores from the 3-first PC identified the same most significant factors (maturity of cheese, dry matter of processed cheese, $\mathrm{pH}$ and the two-factor interaction between $\mathrm{pH}$ and dry matter of processed cheese) as when evaluating the effects for each sensory attribute separately. In cases with several response variables (i.e. sensory attributes) it may be difficult to grasp the information in a full ANOVA on each of them. Therefore, in such cases, ANOVA of the 2-3 first PC's may represent a

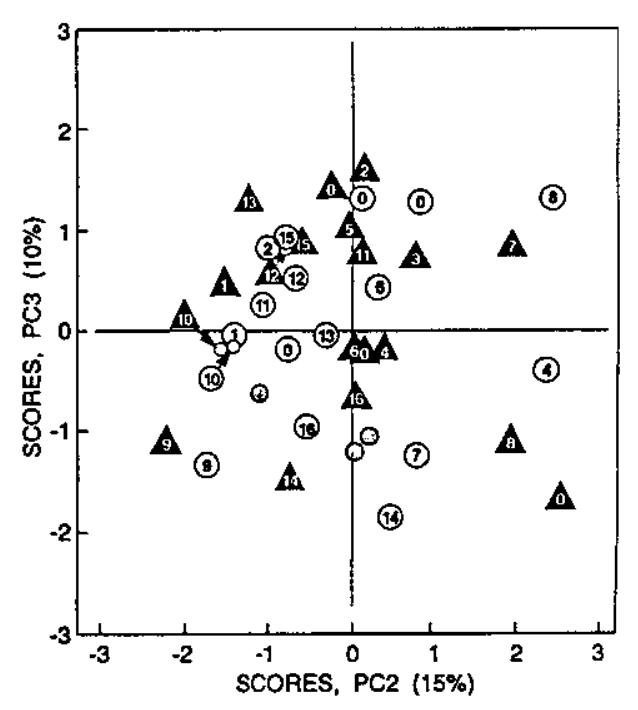

Figure 4a.

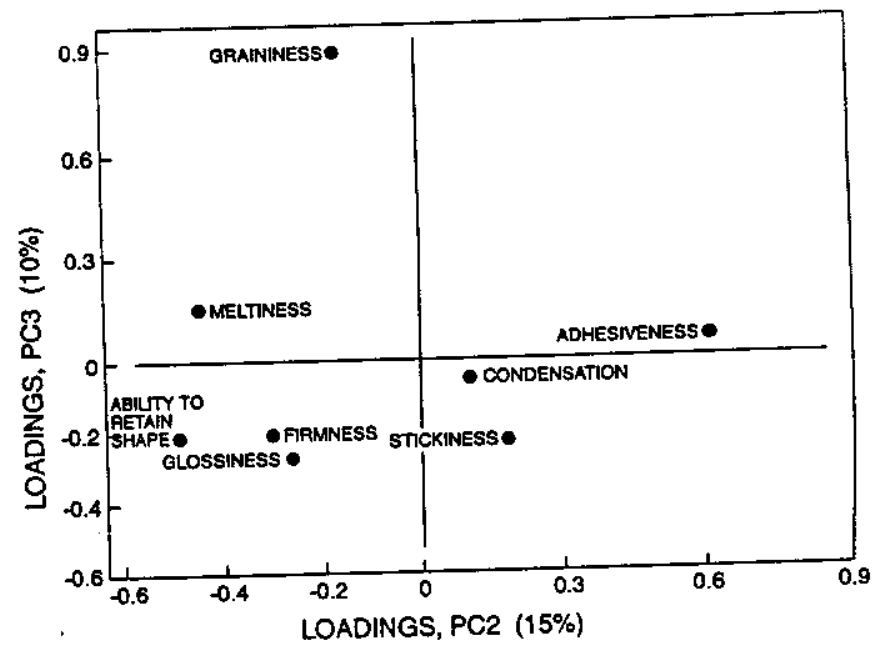

Figure $4 b$.

Figure 4a and 4b. Principal component analysis (PCA) of the sensory data of processed cheese. PCA scores and loadings for principal component 2 and 3 (PC 2 and PC 3). The open circles represent processed cheese produced with melting salt $A$; The triangles represent processed cheese produced with melting salt $B$. Processed cheese produced after today's specifications are indicated by filled circles. 
simplification. The importance of the univariate ANOVA should, however, not be underestimated. It gave valuable and more specific information about the significance for the different factors on each attribute which are not so easily available when using PCA.

A disadvantage of performing ANOVA for each sensory attributes separately is the difficulties of detecting collinearities among the attributes, such collinearities might indicate a 'true' functional dependence between sensory attributes. The sensory attributes describing the main variation among the samples can be evaluated by using the scores from the PC's as response variables. Sensory attributes describing the same phenomena might have high positive or negative loadings at the same $\mathrm{PC}$.

The multivariate approach revealed relationships between samples that were not available when looking at the individual variables separately. For instance, information about closeness to the reference sample as well as information about which samples to prefer according to the criteria set became quite clear when looking at the score and the loading plots. By plotting the scores and loadings for a less significant but still relevant $\mathrm{PC}$ it was possible to identify the samples among the "preferred" which had the lowest intensity of the unwanted properties.

In Figure 5 we have suggested a possible strategy for improving the quality of a product based on the multivariate approach. This is a systematic approach which includes all the steps discussed in this article. Further production of processed cheese can be done according to the new improved processing conditions where it is important to control the processing conditions with greatest effect on the quality, such as grade of maturity, addition of dry matter and $\mathrm{pH}$. Regarding the factors addition of dry matter, after creaming and cooling of processed cheese the levels which are most appropriate and most inexpensive for the company can be chosen as the quality of the processed cheese was minimal affected by the levels used for these factors. For further optimization of the quality of processed cheese the steepest ascent method followed possibly by a central composite design (CCD) (Box et al., 1978) of the most significant factors (maturity of cheese, dry matter content and $\mathrm{pH}$ ) should be performed. It is then possible to identify the processing conditions which results in optimal sensory quality of the processed cheese.

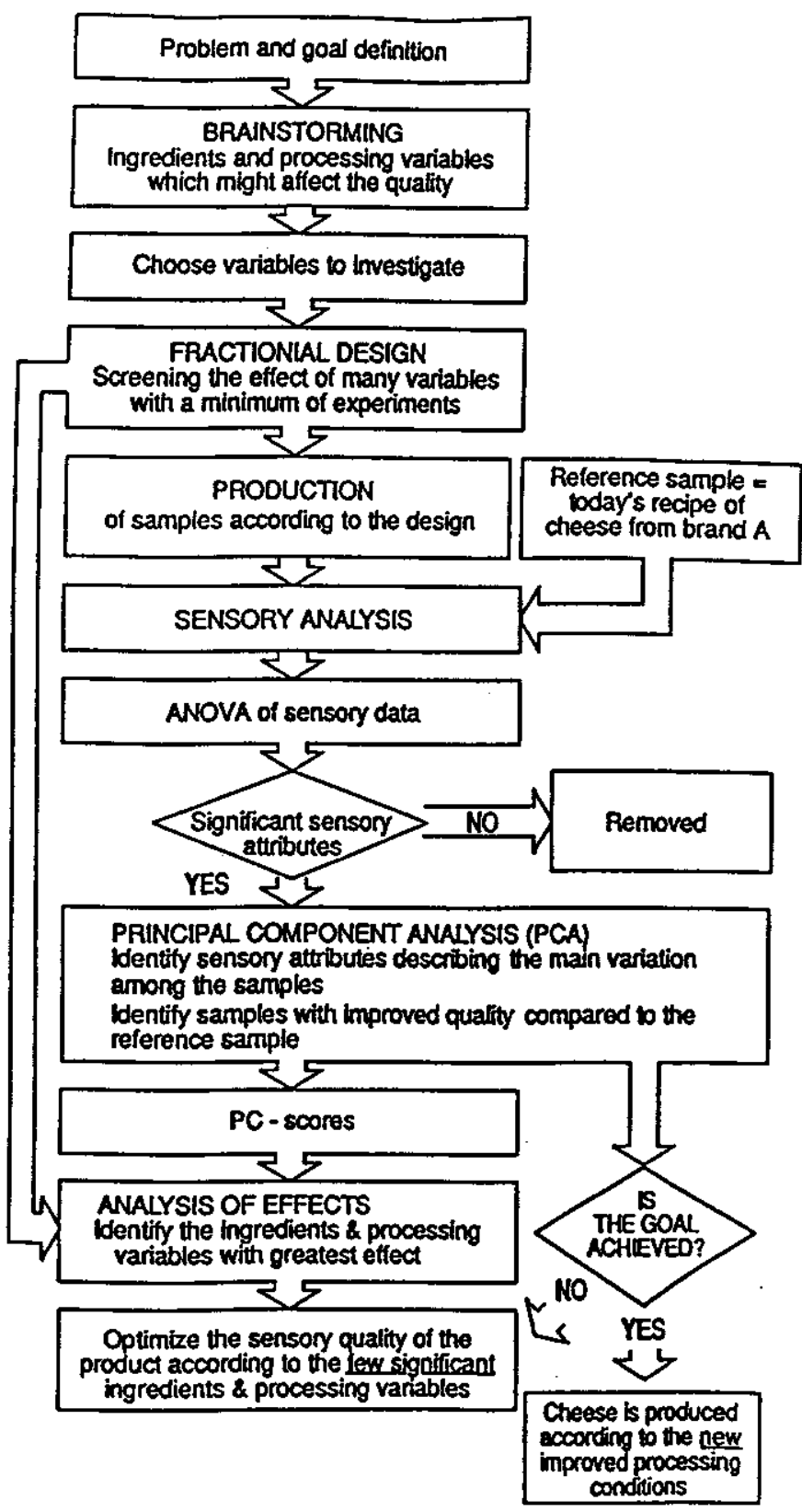

Figure 5. A possible strategy for improving the quality of a product. 


\section{REFERENCES}

Baardseth, P., Naes, T., Mielnik, J., Skrede, G., Hølland, S. \& Eide, O. (1992). "Dairy ingredients effects on sausage sensory properties studied by principal component analysis," $J$. Food Sci., Vol. 57, No. 4. pp. 822-828.

Box, G.E.P., Hunter, W.G. and Hunter, S.J. (1978). Statistics for experimenters. An introduction to Design, Data Analysis and Model Building. John Wiley \& Sons, New York.

Box, G., Bisgaard, S. and Fung, C. (1990). Designing industrial experiments. BBF Books; Madison, WI.

Carlson, R., A. Nordahl, T. Barth and R. Myklebust (1992). "An approach to evaluating screening experiments when several responses are measured," Chemometrics and Intelligent Laboratory systems, Vol. 12. pp. 237-255.

Mardia, K.V., J.T. Kent and J.M. Bibby (1979). Multivariate analysis. Academic Press, London.

Martens, H. and T. Næs (1989). Multivariate calibration. John Wiley \& Sons, Chichester. 